\title{
Short-term effects of catheter-based renal denervation on cardiac sympathetic drive and cardiac baroreflex function in heart failure
}

Lindsea C Booth ${ }^{1}$, Markus P Schlaich ${ }^{2}$, Erika E Nishi ${ }^{1,3}$, Song T Yao ${ }^{1}$, Jianzhong Xu ${ }^{2,4}$, Rohit Ramchandra $^{1,5}$, Gavin W Lambert ${ }^{2}$, Clive N May ${ }^{1}$

${ }^{1}$ Florey Institute of Neuroscience and Mental Health, Melbourne, Australia

${ }^{2}$ Baker IDI Heart and Diabetes Institute and Central Clinical School Monash University, Melbourne, Australia

${ }^{3}$ Department of Physiology, Cardiovascular Division, Federal University of São Paulo, São Paulo, Brazil

${ }^{4}$ Shanghai Institute of Hypertension, Ruijin Hospital, Shanghai, China

${ }^{5}$ Department of Physiology, University of Auckland, Auckland, New Zealand

\section{CORRESPONDENCE TO:}

Dr Lindsea C. Booth,

Florey Institute of Neuroscience and Mental Health,

University of Melbourne, Parkville, Victoria 3010, Australia.

Phone: +61383440182

Fax: $\quad+61393481707$

Email: lindsea.booth@florey.edu.au

\section{Acknowledgement of grant support}

This work was supported by National Health and Medical Research Council of Australia (NHMRC) (1012100) and the Victorian Government's Operational Infrastructure Support Program. L.C. Booth was the recipient of a NHMRC Early Career Fellowship, E.E. Nishi was 
supported by Research Internships Abroad Fellowship of São Paulo Research Foundation, R. Ramchandra was supported by a NHMRC Career Development Fellowship and C.N. May, M.P. Schlaich and G.W. Lambert were supported by NHMRC Research Fellowships.

\section{Any potential conflicts of interest}

C.N. May has received honoraria and travel support for presentations from Medtronic, M.P. Schlaich from Abbott, Servier, Novartis, and Medtronic and G.W. Lambert from Medtronic, Pfizer, Wyeth Pharmaceuticals and Servier. M.P. Schlaich serves on scientific advisory boards for Abbott (formerly Solvay) Pharmaceuticals, Novartis Pharmaceuticals and Medtronic. G.W. Lambert has acted as a consultant for Medtronic. The laboratories of MP Schlaich and G.W. Lambert currently receive research funding from Medtronic, Abbott (formerly Solvay) Pharmaceuticals, Servier Australia and Allergan.

Keywords: Radiofrequency-ablation, Renal denervation, Cardiac sympathetic nerve activity, arterial baroreflex, Animal model 


\section{Abstract}

Objectives: Sympathetic drive, especially to the heart, is elevated in heart failure and is strongly associated with poor outcome. The mechanisms causing the increased sympathetic drive to the heart remain poorly understood. Catheter-based renal denervation (RDN), which reduces blood pressure (BP) and sympathetic drive in hypertensive patients, is a potential treatment in heart failure. The aim of this study was to investigate the short-term effects of catheter-based RDN on BP, heart rate (HR) and cardiac sympathetic nerve activity (CSNA) and on baroreflex function in a conscious, large animal model of heart failure.

Methods: Adult merino ewes were paced into heart failure (ejection fraction $<40 \%$ ) and then instrumented to directly record CSNA. The resting levels and baroreflex control of CSNA and HR were measured before and $24 \mathrm{~h}$ after bilateral renal $(\mathrm{n}=6)$ or sham $(\mathrm{n}=6)$ denervation. RDN was performed using the Symplicity Flex Catheter System ${ }^{\circledR}$ (Medtronic) using the same algorithm as in patients.

Results: Catheter-based RDN significantly reduced resting diastolic $\mathrm{BP}(\mathrm{P}<0.01)$ and mean arterial blood pressure $(\mathrm{P}<0.05)$, but did not change resting HR or CSNA compared with sham denervation. Renal denervation reduced the BP at which CSNA was at $50 \%$ of maximum $(\mathrm{BP} 50 ; \mathrm{P}<0.005)$ compared with sham denervation.

Conclusions: In an ovine model of heart failure, catheter-based RDN did not reduce resting CSNA in the short-term. There was, however, a lack of a reflex increase in CSNA in response to the fall in arterial pressure due to a leftward shift in the baroreflex control of CSNA, which may be due to denervation of renal efferent and/or afferent nerves. 


\section{Introduction}

In heart failure (HF), the inability of the heart to perfuse organs triggers a complex set of compensatory mechanisms, including activation of the renin-angiotensin-aldosterone system (RAAS) and the sympathetic nervous system (SNS) [1]. Sympathetic drive, particularly to the heart as measured by norepinephrine (NE) spillover [2], has been linked to poor outcome [3]. Accordingly, the protective effects of inhibiting branches of RAAS and SNS have been well established in HF [4-8]. Mortality of these patients, however, remains high and, while the benefit of current treatments cannot be underestimated, pharmacotherapy is not without longterm side effects [9].

The role of the sympathetic nerves in cardiovascular disease has gained renewed interest with the introduction of catheter-based renal denervation $(\mathrm{RDN})$ as a treatment in patients with drug resistant hypertension $[10,11]$. Destruction of the renal nerves would be expected to reduce blood pressure considering efferent renal nerves play a major role in renin release, renal vasoconstriction and sodium retention [12]. It is also plausible that increased afferent renal nerve activity may cause a reflex increase in sympathetic outflow and worsening hypertension [13-18]. The possibility of targeting renal nerves to reduce RAAS and SNS activity with a catheter-based approach, therefore, has a lot of appeal as a potential treatment of HF [19]. Indeed, preliminary reports from the first-in-man safety trial in HF patients showed that RDN appeared safe and was associated with an increase in the 6 minute walk distance 6 months after RDN [20]. Importantly for the safety of the procedure, there were no significant reductions in blood pressure following RDN in the seven systolic HF patients [20]. A pilot study has shown that RDN reduces ventricular tachyarrhythmia in HF patients [21] and a larger, randomised controlled trial of renal denervation in HF is ongoing [22].

Studies in experimental models of HF also indicate beneficial effects of RDN on cardiac function; for example, surgical RDN reduced left ventricular filling pressure and improved 
cardiac function following myocardial infarction in rats [23-25] and catheter ablation of renal nerves prior to pacing-induced HF reduced left ventricular filling pressure and the incidence of atrial and ventricular fibrillation in dogs $[26,27]$. In HF rabbits, unilateral surgical RDN prevented the reduction in renal blood flow [28] and improved the reduced heart rate baroreflex sensitivity and decreased plasma NE levels, but did not improve cardiac function [29]. Bilateral RDN attenuated sodium retention following myocardial infarction in rats [30] and in dogs with HF [31]. One of the very few studies investigating RDN in established HF showed improved cardiac and renal function in rats when surgical renal denervation was performed 1 and 4 weeks post-myocardial infarction [25].

Although these studies suggest that RDN has beneficial effects in HF, there are likely to be differences in the responses to surgical and catheter-based RDN, and depending whether RDN is performed pre-HF or in well established HF. The aim of this study was to investigate the short-term effects of bilateral RDN, using the Symplicity Flex system ${ }^{\circledR}$ and the same algorithm used in human patients, on directly recorded cardiac sympathetic nerve activity (CSNA) in pacing-induced ovine HF [32, 33]. 


\section{Methods}

2.1 Surgical procedures. Prior to experiments Merino ewes were individually housed and given free access to water and $800 \mathrm{~g}$ oaten chaff once a day. Anaesthesia was induced $(5 \%)$ and maintained (1.5-2.0\%) with isoflurane- $\mathrm{O}_{2} /$ air mixture. In all surgeries, sheep were treated with intramuscular antibiotics (900 mg, procaine penicillin, Troy Laboratories, NSW) at surgery and 1 day post-operatively. Analgesia was maintained with intramuscular flunixin meglumine (1 mg/kg; Troy Laboratories) at surgery. Experimental procedures were approved by the Animal Ethics Committee of the Florey Institute of Neuroscience and Mental Health under guidelines laid down by the National Health and Medical Research Council of Australia.

Sheep underwent three separate surgical procedures. In the first surgery, sheep were prepared with carotid arterial loops and a pacemaker lead (Medtronic, Minneapolis, MN) was inserted under fluoroscopic guidance into the apex of the right ventricle via the right jugular vein. The lead was exteriorized at the neck, connected to an external pacemaker and HF was induced by rapid ventricular pacing at about 200 beats/min for 8 to 10 weeks. Once sheep were in HF they underwent a second surgery where intra-fascicular recording electrodes were implanted in the cardiac sympathetic nerves [33]. Five days after implantation of electrodes, sheep were anesthetised and a 6F catheter was introduced into the femoral artery and heparin (3000 IU) administered. A Symplicity Flex catheter ${ }^{\circledR}$ (Medtronic Ardian Inc, CA, USA) was introduced into a renal artery under fluoroscopic guidance using radio-contrast and 5-7 two-minute radiofrequency ablations were delivered in a helical configuration along the renal artery, starting as close to the kidney as anatomically possible. The catheter system (Medtronic Ardian Inc) monitored tip impedance and temperature and altered energy delivery in response to an algorithm used in humans [11]. The maximum power delivered during renal ablations was $7 \pm 0 \mathrm{~W}$, reaching maximum temperatures of $57 \pm 5{ }^{\circ} \mathrm{C}$ and resulting in a $-17 \pm 1 \%$ 
change in impedance. Bilateral renal denervation was completed in all 6 sheep. In the sham group $(n=6)$ a $6 \mathrm{~F}$ catheter was inserted and the renal arteries were identified under fluoroscopic guidance, but the denervation catheter was not introduced. A similar amount of radio-contrast was administered in both sham and RDN sheep. The femoral artery puncture wound and skin were sutured and the sheep recovered from anaesthesia.

2.2 Measurement of CSNA and MAP. CSNA was recorded differentially between the pair of electrodes with the best signal-to-noise ratio [33]. The signal was amplified $(x 20,000)$ and filtered (band pass, 300-1,000 Hz), displayed on an oscilloscope, and passed through an audio amplifier and loud speaker. Sympathetic nerve activity $(5,000 \mathrm{~Hz})$ and arterial blood pressure $(100 \mathrm{~Hz})$ were recorded on computer using a CED Micro 1401 interface and Spike 2 software (Cambridge Electronic Design, Cambridge, UK). Data were analyzed on a beat-tobeat basis using custom-written routines in the Spike 2 program. Recordings were made for a 15 min resting period and this level, minus background noise, was defined as the $100 \%$ baseline level. Five minute recordings of CSNA (\%baseline, bursts per 100 heart beats, bursts per minute), MAP and HR were made prior to baroreflex curve generation, described below, pre- and post- RDN or sham denervation.

2.3 Generation of baroreceptor reflex curves. Baroreceptor reflex curves were constructed from data collected during infusions of phenylephrine and sodium nitroprusside, infused in random order [33]. Briefly, phenylephrine and sodium nitroprusside $(67 \mu \mathrm{g} / \mathrm{mL}$ in sterile saline) were infused I.V. at 30,60, 120 and $300 \mathrm{~mL} / \mathrm{min}$ for approximately one minute at each dose resulting in a slow, steady change in blood pressure. In order to construct baroreflex curves, SNA burst size, determined as the number of discriminated spikes between successive diastolic pressures minus background, was divided by heart period [33]. These data were sorted by diastolic blood pressure and averaged in groups of $10 \mathrm{~s}$. To allow data from individual animals to be grouped, spike counts were normalized by calculating the 
percent change in SNA from the mean activity recorded during the 15 min resting period, described above. Percent SNA was plotted against diastolic pressure and regression analysis performed (4-parameter sigmoid relationship; Sigmaplot, Systat Software Inc, San Jose, CA). For generation of the HR baroreflex curve, data were sorted by systolic blood pressure [33].

\subsection{Experimental Protocol}

Development of HF was assessed by weekly measurement of ejection fraction, using short axis M-wave echocardiography in conscious sheep lying on their right side [33]. Following placement of ventricular pacing leads, a basal measurement was made before the start of ventricular pacing, at approximately 200 beats/min. Sheep were considered to be in HF when ejection fraction had fallen to $<40 \%$. Echocardiography and all experiments were conducted with the pacing switched off.

At least three days after surgical implantation of cardiac sympathetic nerve electrodes, a 5min control recording of resting CSNA and arterial pressure was made in conscious HF sheep. Following this, baroreflex curves were generated (described above). The day after control measurements were made, sheep underwent bilateral denervation or the sham procedure. Twenty-four hours after renal denervation, recordings of CSNA and BP were made and a further set of baroreflex curves were generated. Sheep were housed for $4.2 \pm 0.5$ weeks after sham or renal denervation and then euthanized with an intravenous overdose of pentobarbitone $(100 \mathrm{mg} / \mathrm{kg})$ and kidneys taken for NE analysis.

2.5 Plasma renin activity (PRA) assays. In a subset of HF sheep (sham, n=5; RDN, $\mathrm{n}=4$ ), samples were taken for plasma renin activity analysis. Arterial blood $(5 \mathrm{~mL})$ was taken before the intrafascicular recording electrode surgery and 24 hours after RDN/sham denervation. Samples were taken on ice into EDTA tubes, spun at $3000 \mathrm{rpm}$ at $4^{\circ} \mathrm{C}$ and plasma removed and stored at $-80^{\circ} \mathrm{C}$ for analysis and PRA was assayed as previously described [34]. 
2.6 Renal norepinephrine content. Following euthanasia, the kidneys were rapidly removed and $\sim 0.5 \mathrm{~g}$ sections of cortex and medulla were snap frozen in liquid nitrogen. On the day of norepinephrine analysis, kidney samples were thawed and accurately weighed before being homogenized on ice in $0.5 \mathrm{ml}$ of $0.4 \mathrm{M}$ perchloric acid containing $0.01 \%$ EDTA using a glass-glass hand held homogenizer as previously described [35]. The homogenate was then rapidly centrifuged, and the supernatant was collected for subsequent neurochemical analysis. Catecholamines were extracted from the perchloric acid supernatant, with alumina adsorption, separated by high-performance liquid chromatography, and the amounts were quantified by electrochemical detection according to previously described methods [35]. Renal medullary and cortical NE levels were calculated for each animal by averaging samples from the right and left kidneys.

2.7 Statistical Analysis. Statistical analysis was performed in Rcmdr [36, 37]. For cardiovascular variables, baroreflex parameters and PRA, the changes with RDN or sham denervation were calculated and the differences between groups compared using nonparametric Wilcoxon rank sum test. The renal norepinephrine content after RDN or sham denervation were compared using Wilcoxon rank sum test. Statistical significance was accepted when $\mathrm{P}<0.05$. Data are mean \pm standard error of the mean (S.E.). 


\section{Results}

\subsection{Cardiovascular variables}

Prior to RDN or sham denervation, CSNA ( $83 \pm 5$ and $91 \pm 6$ bursts/100 beats, respectively) and HR (90 \pm 5 and $90 \pm 7 \mathrm{bpm}$, respectively) were increased to levels similar to those previously reported in this model [33]. The levels of arterial pressure, HR and CSNA were similar in the treatment and sham groups before denervation. At $24 \mathrm{~h}$ after the procedure there were significantly larger decreases in MAP $(\mathrm{P}<0.05)$ and $\mathrm{dBP}(\mathrm{P}<0.01)$ following $\mathrm{RDN}$ compared with sham denervation (Table 1, Figure 1). Following RDN or sham denervation there was no significant difference between the groups in CSNA, measured as burst incidence (bursts/100 heart beats), burst frequency (bursts/min) or percent of control (Table 1, Figure 1). There was also no difference in HR between the groups after denervation.

\subsection{Baroreflex changes}

Prior to the denervation procedure the CSNA and HR arterial baroreflex curves were similar in the sham and RDN groups (Figure 2 and Table 2). In these groups of sheep with HF, the HR arterial baroreflex curve was desensitised to a similar degree to that seen in our previous publication [33]. Due to differences in the normalisation of CSNA, baroreflex curves were not compared with previous studies.

Following RDN, there was a significant leftward-shift in the CSNA baroreflex curve as demonstrated by the significant decrease in the diastolic blood pressure at half the maximum CSNA (bp50) in the renal denervated compared with the sham denervated group $(\mathrm{P}<0.005$; Table 2). Other variables of the arterial baroreflex control of CSNA, including the top and bottom plateaus, the range and the maximum slope were similar in both groups (Table 2, 
Figure 2). Renal denervation did not cause any significant changes in any variable of the HR arterial baroreflex curve compared with the sham denervated group (Table 2, Figure 2).

\subsection{Plasma renin activity}

In a subset of animals ( $\mathrm{n}=5$ sham denervation, $\mathrm{n}=4$ denervation) PRA was measured before surgery and 24 hours after RDN/sham denervation. After renal denervation PRA decreased from $0.35 \pm 0.11$ to $0.29 \pm 0.11 \mathrm{nmol} / \mathrm{L} / \mathrm{hr}$ (difference of: $-0.10 \pm 0.04$ ) whereas following sham denervation PRA increased from $0.40 \pm 0.08$ to $0.73 \pm 0.09 \mathrm{nmol} / \mathrm{L} / \mathrm{hr}$ (difference of: $0.32 \pm 0.10)$. There was a significant difference between the change in PRA with RDN compared with sham denervation $(\mathrm{P}<0.05)$.

\subsection{Renal Norepinephrine Content}

Four weeks after RDN, NE in the cortex was $1.5 \pm 0.4 \mathrm{pg} / \mathrm{mg}$ tissue and $1.3 \pm 0.4 \mathrm{pg} / \mathrm{mg}$ tissue in the medulla ( $n=6$; Figure 3 ). These levels were significantly lower than in the sham denervated group ( $\mathrm{n}=6$; cortex: $5.3 \pm 0.6 \mathrm{pg} / \mathrm{mg}$ tissue; $\mathrm{P}<0.0001$ and medulla $4.6 \pm 0.7$ $\mathrm{pg} / \mathrm{mg}$ tissue; $\mathrm{P}<0.005)$. 


\section{Discussion}

This study investigated the early changes in sympathetic control of the heart following bilateral renal denervation with the catheter-based Symplicity Flex denervation system in an ovine model of HF. Although RDN did not significantly reduce the resting level of CSNA, it inhibited the baroreflex mediated increase in CSNA in response to the fall in blood pressure following the procedure. This lack of an increase in CSNA resulted from a leftward shift of the CSNA arterial baroreflex curve, which was possibly due to reduced PRA.

Previous experimental studies have found that surgical RDN performed prior to the development of HF has a number of beneficial effects in HF, including improved autonomic balance and baroreflex function, lower incidence of atrial and ventricular fibrillation, reduced sodium retention and increased renal blood flow [23, 24, 26-31]. In addition, improved renal and cardiac function was shown in a study in rats that underwent surgical RDN at 1 and 4 weeks post-myocardial infarction [25]. Although we found a leftward shift in the baroreflex control of CSNA following RDN in our ovine HF model, the effect was relatively minor and there was no significant decrease in the resting levels of CSNA. There are several possible explanations for our findings. The lack of a reduction in CSNA after RDN in our study could be due to (i) the fact that most previous studies have examined the effects of RDN before rather than after the development of $\mathrm{HF}$ as in the present study, (ii) the effect of RDN is predominantly or exclusively directed at the kidneys rather than the heart, or (iii) a reduction in CSNA may require more than 24 hours to take effect, if for example, changes occur after RDN via altered afferent signalling, altered processing within the central nervous system or indeed via vascular changes with loss of efferent outflow. 
The mechanisms by which RDN may improve autonomic function in hypertension and HF are unclear, but denervation of renal afferents and reduced circulating levels of angiotensin II have been proposed as major factors. Although the role of the renal afferents has been studied in the development of hypertension $[38,39]$, there have been few studies that have looked at their role in HF $[40,41]$. This is surprising as the pathology of HF is associated with changes that would be expected to alter afferent renal nerve activity, including increased venous pressure, decreased renal perfusion pressure and blood flow, altered nitric oxide production and increased angiotensin II and endothelin levels [40-44]. In HF, the interaction of these factors with the afferent renal reflex may be more complex; for example, studies in anaesthetised rats indicate that the renal mechanosensory reflex is blunted in HF due to actions of high circulating levels of angiotensin II and endothelin-1 [40, 41]. The lack of a decrease in the resting level of CSNA following RDN in our ovine HF model seems to indicate that in this setting the renal afferents do not play a major role in maintaining the large increase in CSNA. However, as indicated above, it remains to be determined whether CSNA may be reduced at later time points after RDN than those investigated very shortly after the procedure in our study. Similarly, the situation may be different when HF is more severe.

An additional benefit of RDN in HF is that the stimulatory effect of renal SNA on renin release will be removed thus reducing the increased circulating level of angiotensin, which will act to increase SNA. Decreases in plasma renin, Ang II and aldosterone levels have been reported in a small group of HF patients 24 hours after renal nerve blockade under local anaesthesia [45]. In line with these observations, in a subset of our animals we found that RDN caused a decrease in PRA despite a fall in BP, whereas an increase in PRA was observed in the sham group. Of note, the increase in PRA seen with sham denervation is not 
unexpected as previous studies in man have shown increases in PRA 24 hours after general anaesthesia and surgery [46] and radio-contrast administration [47]. Whether this small reduction in PRA, and thus circulating angiotensin II, following RDN is sufficient to account for the leftward shift of baroreflex control of CSNA is unclear, but we have reported similar changes after treatment with irbesartan in this model of HF [48]. Similarly in HF patients treated with enalaprilat, the decrease in blood pressure was not associated with an increase in muscle SNA, as opposed to the increase in muscle SNA seen in control patients [49].

In our study we saw greater reductions in blood pressure (MAP and dBP) in HF animals after RDN compared with sham denervation. Although hypotension is a potential concern following RDN in HF patients, a recent small safety study demonstrated that BP did not change significantly [20]. A major difference between the sheep in this study and the HF patients in the pilot study was that all but one of the HF patients were on either ACEinhibitors or Ang II blockers prior to renal denervation [20]. The lack of any treatment in our sheep would have allowed expression of any hypotensive effect due to a fall in angiotensin levels, which would have been masked in drug treated patients. A significant strength of the current study is that RDN was performed without the confounding effects of concomitant drugs aimed at altering either the RAAS or the sympathetic nervous system.

A problem with catheter-based $\mathrm{RDN}$ is that there is currently no measure of the degree of denervation during the procedure. Using the single electrode Symplicity Flex system the placement of a series of lesions in a helical configuration along a 3-dimensional renal artery is difficult when seen in 2-dimensions on a screen. Despite these technical challenges, we observed significant reductions in renal NE levels approximately one month post-RDN in both the renal medulla and cortex indicating effective, albeit not complete, denervation. 


\section{Strengths and limitations}

The strengths of this study were that denervation was carried out using the same catheter system and algorithm used in human patients in a large animal model of HF, without the confounding effects of treatment with antihypertensive drugs. Furthermore, all cardiovascular changes were measured in conscious animals standing quietly in their home cages, removing any effects of anaesthesia. Direct measurement of CSNA following RDN provides important insights into the effects that RDN may have in HF patients. Importantly, RDN was conducted once sheep were in established HF, the period where human patients would be treated. The limitations of this study were that we did not measure changes in cardiac function or morphology following renal denervation, as in this model of HF the degree of cardiac dysfunction is externally determined by the rate of cardiac pacing. In view of this we did not investigate the cardiovascular changes over several months following RDN; such studies need to be carried out in a myocardial infarction model of HF.

\section{Conclusions}

Our understanding of the detrimental effects of increased SNA in HF, and evidence that this is in part driven by neural afferent reflexes, suggested that RDN may reduce the elevated level of CSNA in HF. In the present study, catheter-based RDN in untreated sheep with pacing-induced HF caused a large reduction in renal NE tissue levels, indicating effective denervation. While RDN did not decrease the high resting level of CSNA, the reflex increase in CSNA in response to the reduction in MAP was abolished due to a leftward shift of the CSNA baroreflex curve. It is possible that the reduction in PRA may have contributed to this shift in the CSNA baroreflex curve since we have found a similar effect with treatment with an AT-1 receptor antagonist in HF [48]. Removal of the afferent renal reflex may also have 
contributed to this shift. These preclinical findings indicate that catheter-based RDN effectively denervates the renal nerves, but does not reduce the damaging high level of CSNA, at least not in the very short term. Further studies are required to determine the longterm effects of RDN on resting CSNA in HF.

\section{Acknowledgements}

The authors acknowledge the expert technical assistance of Anthony Dornom and Alan McDonald in the sheep studies and Sarah Phillips and Dr Nina Eikelis for assistance in catecholamine determination. The authors thank Medtronic who provided the Symplicity Flex RDN System in kind. 


\section{References}

[1] Weiss M, Kenney M, Musch T, Patel K. Modifications to central neural circuitry during heart failure. Acta Physiol Scand. 2003;177:57-67.

[2] Hasking GJ, Esler MD, Jennings GL, Burton D, Johns JA, Korner PI. Norepinephrine spillover to plasma in patients with congestive heart failure: evidence of increased overall and cardiorenal sympathetic nervous activity. Circulation. 1986;73:615-21.

[3] Kaye DM, Lefkovits J, Jennings GL, Bergin P, Broughton A, Esler MD. Adverse consequences of high sympathetic nervous activity in the failing human heart. J Am Coll Cardiol. 1995;26:1257-63.

[4] Kjekshus J, Swedberg K, Snapinn S. Effects of enalapril on long-term mortality in severe congestive heart failure. CONSENSUS Trial Group. The American journal of cardiology. 1992;69:103-7.

[5] A randomized trial of beta-blockade in heart failure. The Cardiac Insufficiency Bisoprolol Study (CIBIS). CIBIS Investigators and Committees. Circulation. 1994;90:1765-73.

[6] Pfeffer MA, Braunwald E, Moye LA, Basta L, Brown EJ, Jr., Cuddy TE, et al. Effect of captopril on mortality and morbidity in patients with left ventricular dysfunction after myocardial infarction. Results of the survival and ventricular enlargement trial. The SAVE Investigators. N Engl J Med. 1992;327:669-77.

[7] Tonkon M, Awan N, Niazi I, Hanley P, Baruch L, Wolf RA, et al. A study of the efficacy and safety of irbesartan in combination with conventional therapy, including ACE inhibitors, in heart failure. Irbesartan Heart Failure Group. International journal of clinical practice. 2000;54:11-4, 6-8.

[8] Ruzicka M, Floras JS, McReynolds AJ, Coletta E, Haddad H, Davies R, et al. Do high doses of AT(1)-receptor blockers attenuate central sympathetic outflow in humans with chronic heart failure? Clinical science (London, England : 1979). 2013;124:589-95.

[9] Bangalore S, Kumar S, Messerli FH. When conventional heart failure therapy is not enough: angiotensin receptor blocker, direct renin inhibitor, or aldosterone antagonist? Congestive heart failure (Greenwich, Conn). 2013;19:107-15.

[10] Esler MD, Krum H, Sobotka PA, Schlaich MP, Schmieder RE, Bohm M. Renal sympathetic denervation in patients with treatment-resistant hypertension (The Symplicity HTN-2 Trial): a randomised controlled trial. Lancet. 2010;376:1903-9.

[11] Krum H, Schlaich M, Whitbourn R, Sobotka PA, Sadowski J, Bartus K, et al. Catheterbased renal sympathetic denervation for resistant hypertension: a multicentre safety and proof-of-principle cohort study. Lancet. 2009;373:1275-81.

[12] DiBona GF, Kopp UC. Neural control of renal function. Physiological reviews. 1997;77:75-197.

[13] Katholi RE, Woods WT. Afferent renal nerves and hypertension. Clinical and experimental hypertension Part A, Theory and practice. 1987;9 Suppl 1:211-26.

[14] Campese VM, Kogosov E, Koss M. Renal afferent denervation prevents the progression of renal disease in the renal ablation model of chronic renal failure in the rat. American journal of kidney diseases : the official journal of the National Kidney Foundation. 1995;26:861-5. 
[15] Schlaich MP, Sobotka PA, Krum H, Lambert E, Esler MD. Renal sympathetic-nerve ablation for uncontrolled hypertension. N Engl J Med. 2009;361:932-4.

[16] Hering D, Lambert EA, Marusic P, Walton AS, Krum H, Lambert GW, et al. Substantial reduction in single sympathetic nerve firing after renal denervation in patients with resistant hypertension. Hypertension. 2013;61:457-64.

[17] Ezzahti M, Moelker A, Friesema EC, van der Linde NA, Krestin GP, van den Meiracker $\mathrm{AH}$. Blood pressure and neurohormonal responses to renal nerve ablation in treatmentresistant hypertension. Journal of hypertension. 2014;32:135-41.

[18] Hering D, Marusic P, Walton AS, Lambert EA, Krum H, Narkiewicz K, et al. Sustained sympathetic and blood pressure reduction 1 year after renal denervation in patients with resistant hypertension. Hypertension. 2014;64:118-24.

[19] Petersson M, Friberg P, Eisenhofer G, Lambert G, Rundqvist B. Long-term outcome in relation to renal sympathetic activity in patients with chronic heart failure. Eur Heart J. 2005;26:906-13.

[20] Davies JE, Manisty CH, Petraco R, Barron AJ, Unsworth B, Mayet J, et al. First-in-man safety evaluation of renal denervation for chronic systolic heart failure: primary outcome from REACH-Pilot study. International journal of cardiology. 2013;162:189-92.

[21] Ukena C, Bauer A, Mahfoud F, Schreieck J, Neuberger HR, Eick C, et al. Renal sympathetic denervation for treatment of electrical storm: first-in-man experience. Clinical research in cardiology : official journal of the German Cardiac Society. 2012;101:63-7.

[22] Verloop WL, Beeftink MM, Nap A, Bots ML, Velthuis BK, Appelman YE, et al. Renal denervation in heart failure with normal left ventricular ejection fraction. Rationale and design of the DIASTOLE (DenervatIon of the renAl Sympathetic nerves in hearT failure with nOrmal Lv Ejection fraction) trial. European journal of heart failure. 2013;15:1429-37.

[23] Nozawa T, Igawa A, Fujii N, Kato B, Yoshida N, Asanoi H, et al. Effects of long-term renal sympathetic denervation on heart failure after myocardial infarction in rats. Heart Vessels. 2002;16:51-6.

[24] Hu J, Li Y, Cheng W, Yang Z, Wang F, Lv P, et al. A comparison of the efficacy of surgical renal denervation and pharmacologic therapies in post-myocardial infarction heart failure. PloS one. 2014;9:e96996.

[25] Hu J, Yan Y, Zhou Q, Ji M, Niu C, Hou Y, et al. Effects of renal denervation on the development of post-myocardial infarction heart failure and cardiac autonomic nervous system in rats. International journal of cardiology. 2014;172:e414-6.

[26] Guo Z, Zhao Q, Deng H, Tang Y, Wang X, Dai Z, et al. Renal sympathetic denervation attenuates the ventricular substrate and electrophysiological remodeling in dogs with pacinginduced heart failure. International journal of cardiology. 2014;175:185-6.

[27] Zhao Q, Yu S, Huang H, Tang Y, Xiao J, Dai Z, et al. Effects of renal sympathetic denervation on the development of atrial fibrillation substrates in dogs with pacing-induced heart failure. International journal of cardiology. 2013;168:1672-3.

[28] Clayton SC, Haack KK, Zucker IH. Renal denervation modulates angiotensin receptor expression in the renal cortex of rabbits with chronic heart failure. Am J Physiol Renal Physiol. 2010;300:F31-9. 
[29] Schiller AM, Haack KK, Pellegrino PR, Curry PL, Zucker IH. Unilateral renal denervation improves autonomic balance in conscious rabbits with chronic heart failure. Am J Physiol Regul Integr Comp Physiol. 2013;305:R886-92.

[30] DiBona GF, Sawin LL. Role of renal nerves in sodium retention of cirrhosis and congestive heart failure. Am J Physiol. 1991;260:R298-305.

[31] Villarreal D, Freeman RH, Johnson RA, Simmons JC. Effects of renal denervation on postprandial sodium excretion in experimental heart failure. Am J Physiol. 1994;266:R1599604.

[32] Ramchandra R, Hood SG, Frithiof R, May CN. Discharge properties of cardiac and renal sympathetic nerves and their impaired responses to changes in blood volume in heart failure. Am J Physiol Regul Integr Comp Physiol. 2009;297:R665-74.

[33] Watson A, Hood S, Ramchandra R, McAllen R, May C. Increased cardiac sympathetic nerve activity in heart failure is not due to desensitization of the arterial baroreflex. Am $\mathrm{J}$ Physiol Heart Circ Physiol. 2007;293:H798-804.

[34] Dunn PJ, Espiner EA. Outpatient screening tests for primary aldosteronism. Australian and New Zealand journal of medicine. 1976;6:131-5.

[35] Lambert GW, Jonsdottir IH. Influence of voluntary exercise on hypothalamic norepinephrine. Journal of applied physiology (Bethesda, Md : 1985). 1998;85:962-6.

[36] Fox J. The R Commander: A Basic Statistics Graphical User Interface to R. J Stat Softw. $2005 ; 14: 1-42$.

[37] R Core Team. R: A language and environment for statistical computing.: R Foundation for Statistical Computing, Vienna, Austria.; 2013.

[38] Oparil S, Sripairojthikoon W, Wyss JM. The renal afferent nerves in the pathogenesis of hypertension. Can J Physiol Pharmacol. 1987;65:1548-58.

[39] Phillips JK. Pathogenesis of hypertension in renal failure: role of the sympathetic nervous system and renal afferents. Clin Exp Pharmacol Physiol. 2005;32:415-8.

[40] Kopp UC, Cicha MZ, Smith LA. Impaired responsiveness of renal mechanosensory nerves in heart failure: role of endogenous angiotensin. Am J Physiol Regul Integr Comp Physiol. 2003;284:R116-24.

[41] Kopp UC, Cicha MZ, Jones SY. Activation of endothelin A receptors contributes to impaired responsiveness of renal mechanosensory nerves in congestive heart failure. Can $\mathrm{J}$ Physiol Pharmacol. 2010;88:622-9.

[42] Ueda H, Uchida Y, Kamisaka K. Mechanism of the reflex depressor effect by the kidney in dog. Jpn Heart J. 1967;8:597-606.

[43] Recordati G, Genovesi S, Cerati D. Renorenal reflexes in the rat elicited upon stimulation of renal chemoreceptors. J Auton Nerv Syst. 1982;6:127-42.

[44] Ashton N, Clarke CG, Eddy DE, Swift FV. Mechanisms involved in the activation of ischemically sensitive, afferent renal nerve mediated reflex increases in hind-limb vascular resistance in the anesthetized rabbit. Can J Physiol Pharmacol. 1994;72:637-43.

[45] Dai QM, Fen Y, Lu J, Ma GS. Efficacy of regional renal nerve blockade in patients with chronic refractory heart failure. Chinese medical journal. 2013;126:1076-80. 
[46] Oyama T, Taniguchi K, Jin T, Satone T, Kudo T. Effects of anaesthesia and surgery on plasma aldosterone concentration and renin activity in man. British journal of anaesthesia. 1979;51:747-52.

[47] Sagy M, Aladjem M, Shem-Tov A, Eshkol A, Orda S, Hegesh J, et al. The renal effects of radiocontrast administration during cardioangiography in two different groups with congenital heart disease. European journal of pediatrics. 1984;141:236-9.

[48] Ramchandra R, Watson AM, Hood SG, May CN. Response of cardiac sympathetic nerve activity to intravenous irbesartan in heart failure. Am J Physiol Regul Integr Comp Physiol. 2010;298:R1056-60.

[49] Dibner-Dunlap ME, Smith ML, Kinugawa T, Thames MD. Enalaprilat augments arterial and cardiopulmonary baroreflex control of sympathetic nerve activity in patients with heart failure. J Am Coll Cardiol. 1996;27:358-64. 
Table 1: Differences pre- and post-bilateral renal denervation (RDN) or sham (Sham-RDN) denervation.

\begin{tabular}{|l|c|c|c|}
\hline Variable & RDN & Sham-RDN & p-value \\
\hline MAP (mmHg) & $-10 \pm 2$ & $-3 \pm 1$ & $0.0298^{*}$ \\
\hline dBP (mmHg) & $-11 \pm 2$ & $-3 \pm 1$ & $0.0086^{* *}$ \\
\hline sBP (mmHg) & $-7 \pm 3$ & $-5 \pm 1$ & NS \\
\hline HR (beats/min) & $6 \pm 5$ & $-4 \pm 4$ & NS \\
\hline CSNA (\% control) & $-8 \pm 10$ & $-18 \pm 6$ & NS \\
\hline CSNA burst frequency (bursts/min) & $1 \pm 5$ & $-4 \pm 5$ & $\mathrm{NS}$ \\
\hline CSNA burst incidence (bursts/100 beats) & $-8 \pm 2$ & $-5 \pm 4$ & $\mathrm{NS}$ \\
\hline PRA (nmol/L/hr) & $-0.10 \pm 0.04$ & $0.32 \pm 0.10$ & $0.0159^{*}$ \\
\hline
\end{tabular}

$* \mathrm{P}<0.05, \quad * * \mathrm{P}<0.01$; Significant differences in the changes following renal denervation compared with sham denervation. Wilcoxon rank sum test. NS: no significant difference. Data are means \pm S.E. 
Table 2: Differences in baroreflex parameters pre- and post-bilateral renal denervation (RDN) or sham denervation (Sham-RDN).

\begin{tabular}{|l|c|c|c|}
\hline \multicolumn{4}{|c}{ Baroreflex control of HR } \\
\hline Variable & RDN & Sham-RDN & p-value \\
\hline Range (bpm) & $2 \pm 6$ & $12 \pm 11$ & NS \\
\hline Max gain & $-0.3 \pm 0.3$ & $0.6 \pm 0.5$ & NS \\
\hline BP50 (mmHg) & $-9 \pm 2$ & $-11 \pm 3$ & NS \\
\hline $\begin{array}{l}\text { Lower plateau } \\
\text { (bpm) }\end{array}$ & $-1 \pm 5$ & $-5 \pm 4$ & NS \\
\hline & Baroreflex control of CSNA & p-value \\
\hline $\begin{array}{l}\text { Variable } \\
\text { (\%ange }\end{array}$ & RDN & Sham-RDN & NS \\
\hline Max gain & $21 \pm 25$ & $20 \pm 27$ & NS \\
\hline BP50 (mmHg) & $-0.1 \pm 1.4$ & $1.1 \pm 0.7$ & NS \\
\hline $\begin{array}{l}\text { Lower plateau } \\
\text { (\%baseline) }\end{array}$ & $-1 \pm 5$ & $-7 \pm 1$ & $1 \pm 2$ \\
\hline
\end{tabular}

${ }^{\#} \mathrm{P}<0.005$; Wilcoxon rank sum test of difference between pre and post denervation $v$ s. pre and post sham denervation. $\mathrm{n}=6$ per group. NS: no significant difference. Data are means \pm S.E. 


\section{Figure legends}

Figure 1: Cardiovascular changes pre- and post-renal denervation (black symbols) and sham denervation (grey symbols) in sheep with heart failure. Mean arterial blood pressure (MAP), heart rate (HR), cardiac sympathetic nerve activity (CSNA) expressed as \% of baseline, burst frequency and burst incidence. Data points are individual points and 5 min averages \pm S.E.; $\mathrm{n}=6$ in each group.

Figure 2: Baroreflex curves for heart rate (HR) against systolic blood pressure (sBP) and cardiac sympathetic nerve activity (CSNA) against diastolic BP (dBP) before (solid lines) and after (dashed lines) bilateral renal denervation (black symbols) and sham denervation (grey symbols). Symbols represent mean resting points \pm S.E.

Figure 3: Norepinephrine content of the renal cortex (A; ng/g tissue) and renal medulla (B; $\mathrm{ng} / \mathrm{g}$ tissue) following renal denervation (black bars) or sham denervation (grey bars). Data points are averaged left and right kidneys from 6 sheep in each group. $* \mathrm{P}<0.005,{ }^{*} * \mathrm{P}<0.0001$ compared to sham denervation. 
Figure 1:
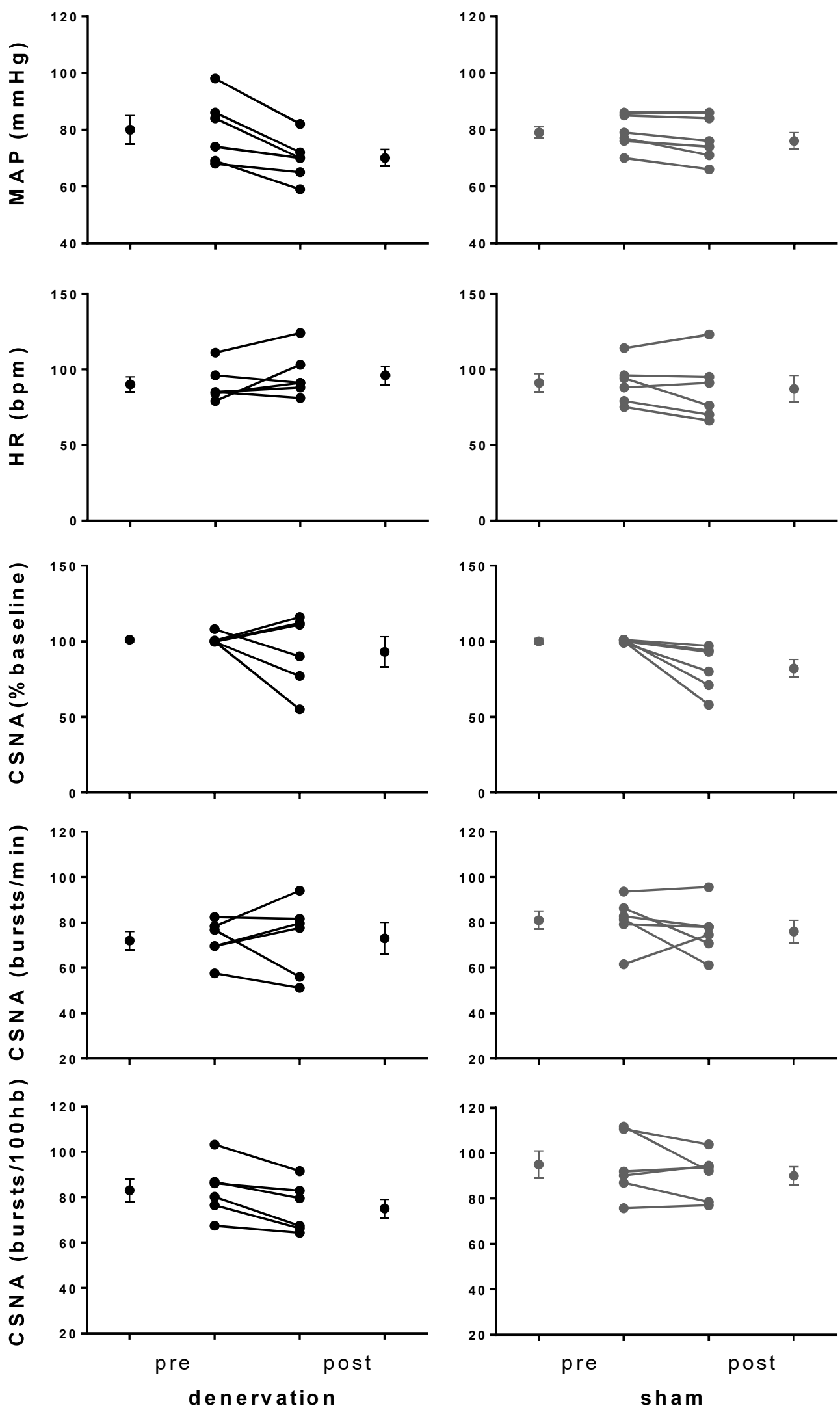
Figure 2:
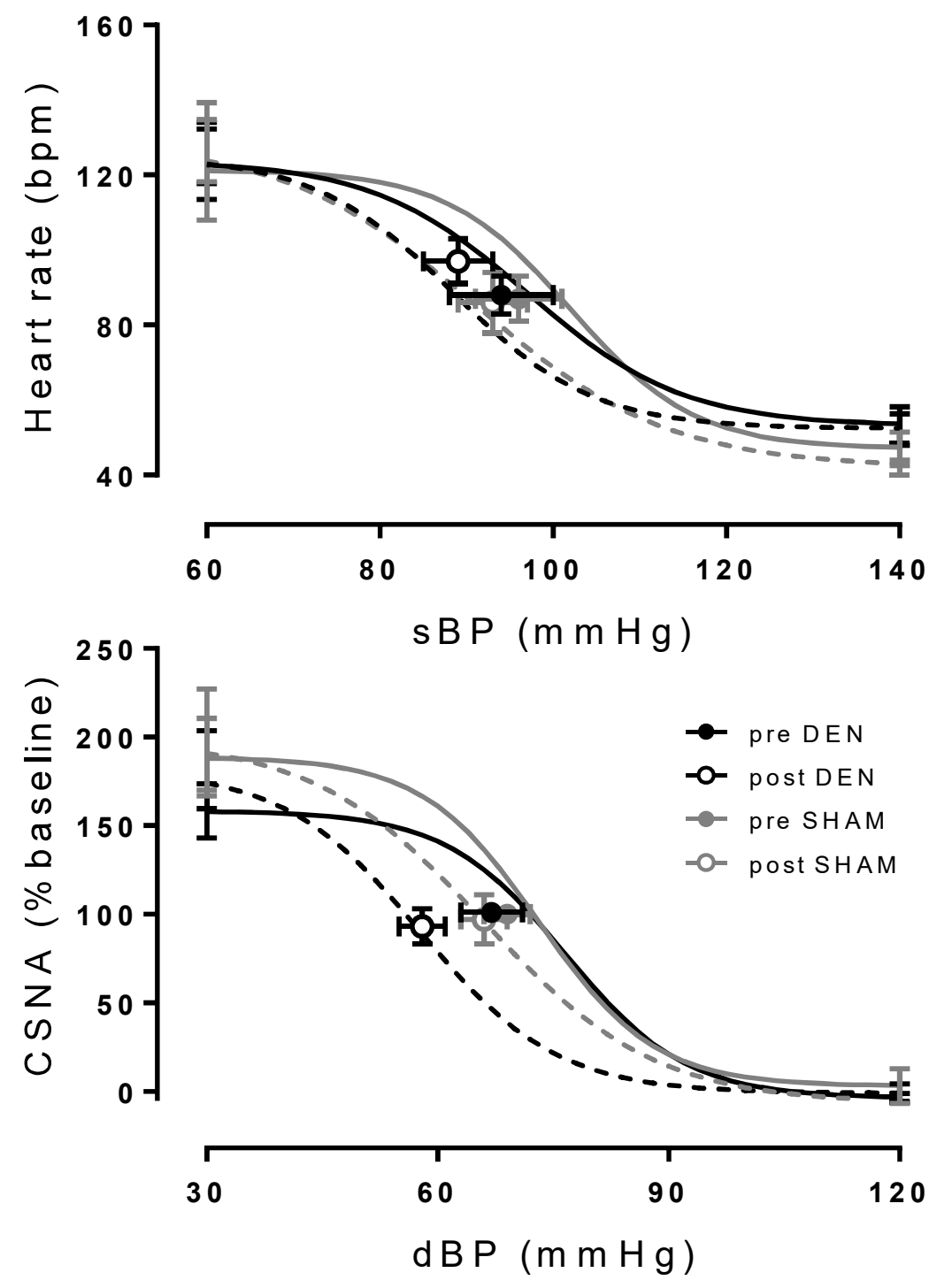
Figure 3:
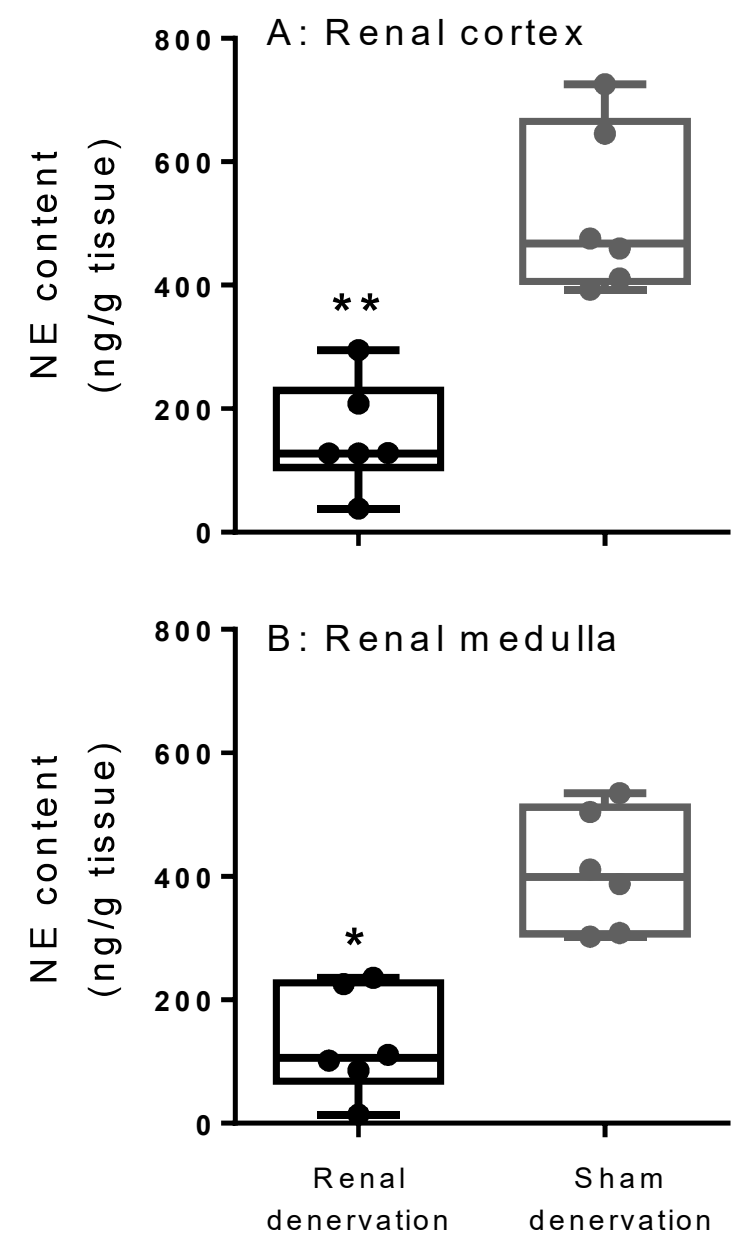